\title{
Purification and Partial Characterization of a Megakaryocyte Colony-stimulating Factor from Human Plasma
}

\author{
Ronald Hoffman, Hsin Hsin Yang, Edward Bruno, and John E. Straneva \\ Indiana Elks Cancer Research Center and Hematology/Oncology Section, Department of Medicine, \\ Indiana University School of Medicine, Indianapolis, Indiana 46223
}

\begin{abstract}
Human plasma obtained from patients with hypomegakaryocytic thrombocytopenia contains a factor that promotes megakaryocyte colony formation by normal human marrow cells. This megakaryocyte colony-stimulating factor was purified from such a plasma specimen. A four-step purification scheme which included ammonium sulfate precipitation, diethylaminoethylSepharose chromatography, affinity chromatography on wheat germ lectin-Sepharose $6 \mathrm{MB}$, and reverse-phase high performance liquid chromatography resulted in a recovery of $16.6 \%$ of the initial biological activity and an increase in specific activity by 3,489 -fold. The purified protein produced a single band on sodium dodecyl sulfate-polyacrylamide gel electrophoresis. Purified megakaryocyte colony-stimulating factor was capable of promoting megakaryocyte colony formation at a concentration of $7.6 \times 10^{-8} \mathrm{M}$. Megakaryocyte colony-stimulating factor was shown to be a glycoprotein and had an apparent 46,000 mol wt. Deglycosylation of megakaryocyte colony-stimulating factor by treatment with trifluoromethanesulfonate resulted in the loss of its ability to promote megakaryocyte colony formation. Megakaryocyte colony-stimulating factor appears to be an important regulator of in vitro human megakaryocytopoiesis at the level of the colony-forming unit megakaryocyte and may be of importance physiologically.
\end{abstract}

\section{Introduction}

Megakaryocytopoiesis appears to be regulated by two factors that specifically affect the proliferation of progenitor cells and the development of megakaryocytes in vitro $(1,2)$. In this scheme, megakaryocyte colony-stimulating factor (Meg-CSF) ${ }^{1}$ modulates the proliferation of the progenitor cell pool, whereas

This work has appeared in abstract form (1984. Clin. Res. 32:496A). Received for publication 4 May 1984 and in revised form 17 September 1984.

1. Abbreviations used in this paper: BFU-E, erythroid burst-forming unit; CFU-E, erythroid colony-forming unit; CFU-GM, granulocytemacrophage colony-forming unit; CFU-M, megakaryocyte colonyforming unit; Epo, erythropoietin; GM-CSA, granulocyte-macrophage colony-stimulating activity; Meg-CSF, megakaryocyte colony-stimulating factor; PAGE, polyacrylamide gel electrophoresis; PAS, periodic acidSchiff; PGP, (human) platelet glycoproteins; PHA-LCM, phytohemagglutinin-stimulated leukocyte-conditioned media; TFMSA, trifluoromethane sulfonate; TSF, thrombopoietic stimulating factor or thrombopoietin.

J. Clin. Invest.

(c) The American Society for Clinical Investigation, Inc. 0021-9738/85/04/1174/09 \$1.00

Volume 75, April 1985, 1174-1182 thrombopoietin influences megakaryocyte development (2). Similar dual requirements for hematopoietic regulatory molecules have been described in the erythroid (3) and macrophage pathways (4).

Urine $(5,6)$, serum $(7)$, and plasma $(8,9)$ obtained from patients with hypomegakaryocytic thrombocytopenia have been shown to promote the formation of megakaryocyte colonies by marrow mononuclear cells in vitro. Phytohemagglutininstimulated leukocyte-conditioned media as well as normal bone and lung conditioned media contain a similar activity (10). Recently Messner et al. (8) have suggested that human plasma is superior to serum in its ability to support megakaryocyte colony formation in vitro. Kimura et al. (9) have demonstrated that factors released from platelets during the clotting process inhibit megakaryocyte colony formation. Vainchenker et al. (11) also suggest that there are substances present in human serum which are essential for megakaryocyte colony formation, and that an additional factor is present which is capable of inhibiting colony formation. The exact role of this inhibitory substance in the regulation of human megakaryocytopoiesis is unclear.

Previous studies of in vitro megakaryocytopoiesis have utilized unfractionated conditioned media (8), serum (7), or plasma (8) as sources of Meg-CSF. In the present report, we describe the purification and partial characterization of MegCSF from human plasma. The availability of this purified molecule will help to delineate the complex cellular and molecular events that occur during human megakaryocytopoiesis.

\section{Methods}

\section{Source of Meg-CSF}

Meg-CSF was purified from plasma obtained from a 36-yr-old white woman with an acquired hematologic disorder characterized by macrocytosis (mean corpuscular volume, $124 \mathrm{fl}$ ), anemia (packed cell volume, $22 \%$ ), mild leukopenia, and severe thrombocytopenia (platelet count $<10,000 \mathrm{~mm}^{3}$ ). Bone marrow specimens obtained on several occasions were noted to be normocellular with megaloblastoid erythroid maturation and normal granulocyte development. The marrow specimens were most notable for a total absence of megakaryocytic elements. This clinical picture was compatible with a diagnosis of selective hypomegakaryocytic thrombocytopenia (12). Because the hematopoietic failure state might have been due to an antibody which was cytotoxic to megakaryocytic progenitor cells (13), the patient was plasmapheresed on 5, 7, and 10 August 1981. This procedure resulted in no hematologic improvement. On each occasion 3 liters of plasma were removed and replaced by an equivalent volume of normal saline, $5 \%$ albumin, and fresh frozen plasma (1:1:1). The plasma obtained after plasmapheresis was sterilized by ultrafiltration, heat inactivated at $56^{\circ} \mathrm{C}$ for $30 \mathrm{~min}$, pooled, and stored at $-80^{\circ} \mathrm{C}$. The plasma was a gift of Dr. C. D. Spencer, Malcolm Grow U. S. Air Force Medical Center, Andrews Air Force Base, Washington, DC. 


\section{Reagents}

Ammonium sulfate, Tris base, Tris- $\mathrm{HCl}$, and $\mathrm{N}$-acetyl-D-glucosamine were purchased from Sigma Chemical Co. (St. Louis, MO). Acrylamide, bisacrylamide, sodium dodecyl sulfate (SDS), and other reagents for electrophoresis were purchased from Bio-Rad Laboratories (Richmond, CA). Trifluoromethane sulfonate (TFMSA) and acetonitrile were obtained from Aldrich Chemical Co. (Milwaukee, WI). DEAE-Sepharose CL-6B and wheat germ lectin Sepharose 6MB were obtained from Pharmacia Fine Chemicals (Piscataway, NJ). Human urinary erythropoietin (Epo) with a specific activity of not $<500 \mathrm{U} / \mathrm{mg}$ protein was obtained from Alpha Therapeutic Corp. (Los Angeles, CA), Lot no. EH009T0. Other chemicals were reagent grade. Double-deionized water was used throughout the studies.

\section{Meg-CSF assay}

Meg-CSF activity was assayed in plasma clot cultures containing 5 $\times 10^{5}$ bone marrow mononuclear cells (14). Bone marrow aspirations were obtained, after informed consent, from the posterior iliac crest under local anesthesia from hematologically normal paid volunteers. The bone marrow aspirate was diluted 1:1 with $\alpha$-medium minus nucleosides (Gibco Laboratories, Grand Island, NY), containing preservative-free sodium heparin at $20 \mathrm{U} / \mathrm{ml}$ and layered over an equal volume of Ficoll-Paque (sp gr of $1.077 \mathrm{~g} / \mathrm{cm}^{3}$; Pharmacia Fine Chemicals). Density centrifugation was performed at $500 \mathrm{~g}$ for $25 \mathrm{~min}$ at $4^{\circ} \mathrm{C}$ in a Beckman model J-6B centrifuge (Beckman Instruments, Inc., Fullerton, CA). The interface mononuclear cell layer was collected and washed with $\alpha$-medium minus nucleosides containing $2 \%$ fetal calf serum. Mononuclear cells at $5 \times 10^{5}$ cells $/ \mathrm{ml}$ were cultured in $1-\mathrm{ml}$ $\mathrm{vol}$ in 35-mm petri dishes. The plasma clot technique of McLeod et al. (15) was modified by the substitution of heat-inactivated human $\mathrm{AB}$ serum for fetal calf serum, and $\alpha$-medium minus nucleosides for NCTC-109 medium and Eagle's minimal essential medium with Hanks' balanced salt solution. Samples to be assayed for Meg-CSF activity were diluted in $\alpha$-medium minus nucleosides at several dilutions. The final $1-\mathrm{cm}^{3}$ aliquot of each culture contained the following supplements: minimal essential medium, nonessential amino acids $(0.02 \mathrm{mmol} / \mathrm{ml})$, L-glutamine $(0.4 \mathrm{mmol} / \mathrm{ml})$, and sodium pyruvate $(0.2 \mathrm{mmol} / \mathrm{ml})$. Culture dishes were incubated for $10-12 \mathrm{~d}$ (unless otherwise stated) at $37^{\circ} \mathrm{C}$ in a $100 \%$ humidified atmosphere of $5 \%$ $\mathrm{CO}_{2}$ in air. Harvesting was performed by in situ fixation with methanol: acetone (1:3) for $20 \mathrm{~min}$, washed with $0.01 \mathrm{M}$ phosphate-buffered saline (PBS), pH 7.2, distilled water, and then air-dried. Plasma clots were stored frozen at $-20^{\circ} \mathrm{C}$ until immunofluorescent staining was performed.

\section{Preparation of antiplatelet glycoprotein antiserum}

Antiplatelet glycoprotein antiserum was utilized to identify megakaryocyte colonies. Purified human platelet glycoprotein (PGP) was prepared by lithium diiodosalicylate-phenol extraction of pooled human platelet concentrates as described by Marchesi and Chasis (16). New Zealand White rabbits were initially immunized by subcutaneous injections of $1 \mathrm{mg}$ of PGP in Freund's complete adjuvant, followed by intramuscular injections ( $1 \mathrm{mg}$ of PGP in Freund's incomplete adjuvant) at 2 and $4 \mathrm{wk}$. Serum was harvested at $6 \mathrm{wk}$ by cardiac puncture and stored in aliquots at $-80^{\circ} \mathrm{C}$. The specificity of the rabbit anti-PGP antiserum has been previously established (17). This antiserum has been found to precipitate $80 \%$ of radiolabeled lithium diiodosalicylateextracted PGP, but nonimmune rabbit serum precipitated only $15 \%$ of the radioactivity. Slab gel electrophoresis of immunoprecipitates corresponded to PGP of estimated mol wt of $145,000,125,000$, and 95,000 , demonstrating activity of the antiserum for the original immunogen (17). When normal bone marrow smears were examined by using indirect immunofluorescent labeling, only platelets, megakaryocytes, and an infrequent population of lymphoidlike cells fluoresced intensively (17). These lymphoidlike cells are thought to represent immature megakaryocytic elements. Preadsorption of the antiserum through a PGP-Sepharose affinity column has been reported to remove all platelet- and megakaryocyte-labeling activity (17).

\section{Immunofluorescent identification of human megakaryocyte colonies}

Whole rabbit anti-PGP antiserum was diluted in PBS (1:200), layered over the fixed plasma clot culture, and incubated for $60 \mathrm{~min}$ at room temperature in $100 \%$ humidified air. After washing three times with PBS, the specimens were reincubated for an additional $60 \mathrm{~min}$ with fluorescein-conjugated goat anti-rabbit IgG (Meloy Laboratories, Inc., Springfield, VA) diluted in PBS, final concentration $0.36 \mathrm{mg}$ of protein/ $\mathrm{ml}$. After washing with PBS, the specimens were counterstained with $0.125 \%$ Evans's Blue for $1.5 \mathrm{~min}$, washed with distilled water, and mounted in isotonic barbital buffer, $\mathrm{pH} 8.6$, in glycerol (1:3).

Plasma clot cultures were scored in situ to enumerate fluoresceinpositive colonies. The 35-mm petri dishes were inverted, and the base area was completely scanned with a fluorescence microscope at $\times 100$ (Zeiss standard microscope 18 with IV FL vertical fluorescent illuminator; Carl Zeiss, Inc., New York). A megakaryocyte colony was defined as a cluster of three or more intensely fluorescent cells. Each study was performed in duplicate to quadruplicate. Specific activity of Meg-CSF preparations was calculated from the number of megakaryocyte colonies per milligram of protein from the linear portion of the dose-response curve.

\section{Assays for other hematopoietic colonies}

Both erythroid burst-forming unit (BFU-E)- and erythroid colonyforming unit (CFU-E)-derived colony assays were performed in plasma clot cultures as previously described (18). Urinary sheep Epo, $11 \mathrm{IU} /$ $\mathrm{mg}$ of protein (Hyclone Tissue Culture Products, Sterile Systems, Inc., Logan, UT, Lot no. 10004) was utilized in these studies. Plasma clots were fixed in situ with $5 \%$ glutaraldehyde in $0.01 \mathrm{M}$ phosphate buffer (pH 7.0-7.2). CFU-E- and BFU-E-derived colonies were harvested after 7 and 12-14 d of incubation, respectively. Cultures were stained with $1 \%$ benzidine and hematoxylin as previously described (19). Maximum growth of CFU-E- and BFU-E-derived colonies in this modified plasma clot assay system was seen at Epo concentrations of 0.5 and $1.0 \mathrm{IU} / \mathrm{ml}$, respectively.

The colony assay for granulocyte-macrophage colony-forming units (CFU-GM) was carried out according to the method of Fauser and Messner (20). Marrow mononuclear cells were suspended in $35-\mathrm{mm}$ Lux standard tissue culture dishes, containing a $1-\mathrm{ml}$ mixture of Iscove's modified Dulbecco's medium (IMDM; Gibco Laboratories), $0.8 \%$ methylcellulose, $30 \%$ fetal bovine serum, $5 \%$ medium conditioned by leukocytes from patients with hemochromatosis in the presence of $1 \%$ phytohemagglutinin (PHA-LCM) $(21)$, and $5 \times 10^{-5} \mathrm{M} 2$-mercaptoethanol. Dishes were incubated at $37^{\circ} \mathrm{C}$ in a humidified atmosphere flushed with $5 \% \mathrm{CO}_{2}$ in air. Colonies were scored with an inverted microscope after 13-14 d of incubation. Clusters of $\geq 40$ cells, many of which had typical band or segmented polymorphonuclear leukocyte morphology were defined as CFU-GM-derived colonies.

\section{Protein estimations}

Protein was estimated by the method of Lowry et al. (22) using bovine serum albumin as a standard. The absorbance at $280 \mathrm{~nm}$ was used to monitor the relative protein concentrations of column effluents.

\section{Purification of Meg-CSF}

Ammonium sulfate fractionation. To $400 \mathrm{ml}$ of plasma, $\left(\mathrm{NH}_{4}\right)_{2} \mathrm{SO}_{4}$ (116.4 g; 50\% saturation) was added with stirring. The solution was kept at $4^{\circ} \mathrm{C}$ for $30 \mathrm{~min}$ and then centrifuged at $27,000 \mathrm{~g}$ at $4^{\circ} \mathrm{C}$ for $20 \mathrm{~min}$ in a refrigerated centrifuge (J2-21 model, Beckman Instruments, Inc., Palo Alto, CA). The supernatant was decanted and the sediment was discarded. The supernatant was subsequently sequentially adjusted to 60,70 , and $80 \%$ saturation by the addition of $\left(\mathrm{NH}_{4}\right)_{2} \mathrm{SO}_{4}$ while the mixture was stirred and the centrifugation cycle was repeated as 
described above. The sediments from each saturation level were resuspended in $0.15 \mathrm{M} \mathrm{NaCl}$ and dialyzed against $0.01 \mathrm{M} \mathrm{K}_{2} \mathrm{HPO}_{4}$

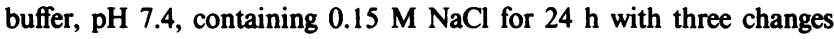
of the dialysate every $8 \mathrm{~h}$. This was followed by two additional changes of the dialysate with distilled water at 12 -h intervals at $4^{\circ} \mathrm{C}$. These samples, which were the sediments produced by the 60,70 , and $80 \%$ $\left(\mathrm{NH}_{4}\right)_{2} \mathrm{SO}_{4}$ fractionation steps were lyophilized and stored at $-20^{\circ} \mathrm{C}$. Each of these fractions was subsequently dissolved in $\alpha$-media and assayed for Meg-CSF activity.

Ion exchange column chromatography. A C10/40 column (Pharmacia Fine Chemicals) was packed with DEAE-Sepharose CL-6B previously equilibrated with $0.05 \mathrm{M}$ sodium acetate buffer, $\mathrm{pH}$ 5.4, containing $0.02 \% \mathrm{NaN}_{3}$ (starting buffer). The lyophilized $\left(\mathrm{NH}_{4}\right)_{2} \mathrm{SO}_{4}$ precipitates were individually dissolved in starting buffer and sequentially applied to the column at a flow rate of $15 \mathrm{ml} / \mathrm{h}$. The column was subsequently eluted with the following elution buffers: (a) $0.05 \mathrm{M}$ sodium acetate buffer, $\mathrm{pH}$ 5.4; (b) $0.05 \mathrm{M}$ sodium acetate buffer containing $0.15 \mathrm{M} \mathrm{NaCl}$; and (c) $0.05 \mathrm{M}$ sodium acetate buffer containing $1 \mathrm{M} \mathrm{NaCl}$, at a flow rate of $15 \mathrm{ml} / \mathrm{h} .130$ fractions $(4 \mathrm{ml}$ each) were collected. Column effluent was monitored with a Gilson absorbance monitor (Gilson Co., Inc., Worthington, $\mathrm{OH}$ ) at $A_{280}$ with chart recorder (Fig. 1). Fractions were pooled, dialyzed against sterile water, and tested for Meg-CSF activity. Active fractions were lyophilized and stored at $-20^{\circ} \mathrm{C}$.

Wheat germ lectin affinity chromatography. Wheat germ lectin was coupled to Sepharose beads after cyanogen bromide activation as described by the method of March et al. (23). An active fraction (fraction V) obtained after ion exchange chromatography (Fig. 1, Table I) was dissolved in $0.05 \mathrm{M} \mathrm{Na}_{2} \mathrm{HPO}_{4}$ buffer, $\mathrm{pH} 7.2$ (PBS) containing $0.02 \% \mathrm{NaN}_{3}$ and applied to a column (K9/15, Pharmacia Fine Chemicals), which was packed with cyanogen bromide-activated wheat germ lectin-Sepharose beads. The column was preequilibrated and desorbed with PBS, pH 7.2, containing $0.2 \mathrm{M} \mathrm{NaCl}$. Buffer was applied to the column by a peristaltic pump, the flow rate was adjusted to 25 $\mathrm{ml} / \mathrm{h}$, and $1.5-\mathrm{ml}$ fractions were collected. Protein in the column effluent was monitored by a Gilson absorbance monitor (Gilson Co., Inc.) at $A_{280}$ with chart recorder. The column was washed with PBS until the effluent was free of protein. The column was subsequently batch eluted with $0.1 \mathrm{M} N$-acetyl-D-glucosamine (Fig. 2). Column fractions were pooled according to the absorbance at $A_{280}$ (Fig. 2). Free sugar and salts were removed by gel filtration with a Bio-Gel P-6DG column (Bio-Rad Laboratories). Lyophilized active fractions were stored at $-20^{\circ} \mathrm{C}$.

Reverse-phase high-performance liquid chromatography. The freezedried fraction $(15 \mu \mathrm{g})$ from the wheat germ lectin-affinity chromatography step (Fig. 3) was dissolved in $30-10 \% \mathrm{H}_{2} \mathrm{O} / \mathrm{CH}_{3} \mathrm{CN}$. The mixture was injected onto a Whatman C18 ODS3 column (Whatman Inc., Clifton, NJ) $(0.4 \times 25 \mathrm{~cm})$. The column was eluted at $0.5 \mathrm{ml} / \mathrm{min}$ with a linear gradient of $30-70 \%$ acetonitrile. The column effluent was monitored by absorbance at $270 \mathrm{nM}$ and recorded. To remove acetonitrile, fractions were diluted with 3 vol of water and dried with a Savant Speed Vac concentrator (Savant Instruments, Inc., Hicksville, $\mathrm{NY})$. These fractions were stored at $-20^{\circ} \mathrm{C}$.

Recovery of biologically active factor from SDS-polyacrylamide gel electrophoresis (PAGE) gel. Samples $(2 \mathrm{ml})$ of concentrated fractions obtained following wheat germ lectin-Sepharose CL-6B chromatography were run on slab gels $\left(3 \times 140 \times 110 \mathrm{~mm}^{3}\right)$ together with standard molecular weight markers. At the end of the run the distance from the tracking dye to the top of the running gel was carefully measured. A strip of the gel was cut vertically for staining and the other part was retained for measurement of biological activity. The unstained part of the gel was sliced horizontally in 5-mm sections according to the position of bands on the stained gel. The gel slices were shaken overnight at $4^{\circ} \mathrm{C}$ in $10 \mathrm{ml}$ of 0.02 sodium phosphate buffer, pH 7.2. The buffer solution was decanted and saved. This extraction step was repeated once and the gel slices then removed and discarded. These supernatants were combined and centrifuged to further remove gel particles and were then dialyzed against double-distilled water for 24 h with three changes of water, followed by ultrafiltration using an Amicon model 52 cell concentrator (Amicon Corp., Lexington, MA), lyophilized, and stored at $-20^{\circ} \mathrm{C}$ until it was reconstituted in $\alpha$-media for use in the biological assay. The strip part of the gel was fixed, washed, and stained as a template. When comparing the unstained portion of the gel with the biologically assayed portion, the gel swelling was taken into account.

$P A G E$. The partially purified fractions were analyzed by electrophoresis in the presence of SDS according to the method of Laemmli (24) (Fig. 4). Samples were electrophoresed on 7.5 or $10 \%$ acrylamide

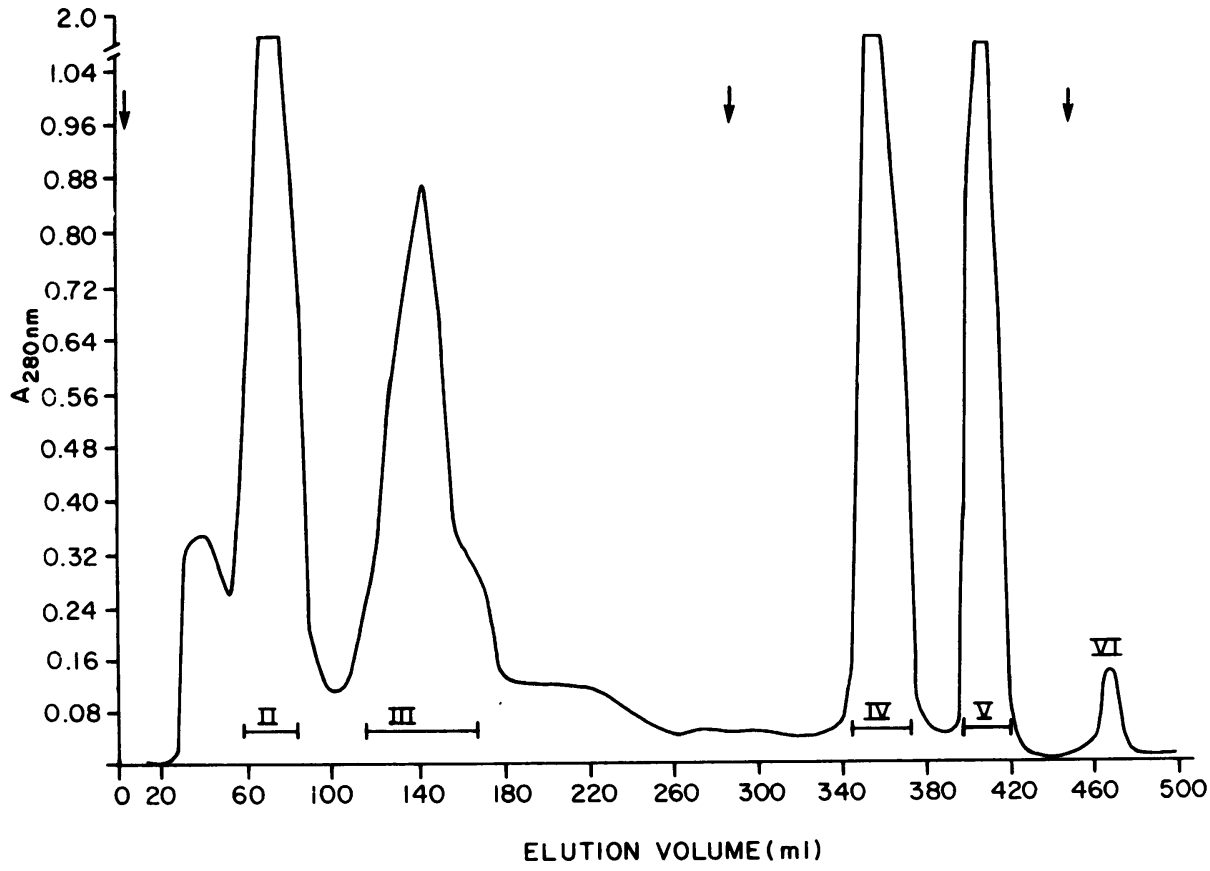

Figure 1. Elution pattern resulting from ion exchange column chromatography on DEAE-Sepharose CL$6 \mathrm{~B}$ of the $80 \%$ saturated ammonium sulfate fraction of human hypomegakaryocytic thrombocytopenic plasma. The column was equilibrated with $0.05 \mathrm{M} \mathrm{NaOAc}$ buffer, pH 5.4 (starting buffer). The protein sample, $1 \mathrm{~g} / 5 \mathrm{ml}$ of starting buffer, was applied and the absorbance monitored at $280 \mathrm{~nm}$ at $4^{\circ} \mathrm{C}$. The arrows indicate the buffer changes as specified in Methods. The Roman numerals indicate pools made for assay. 
Table I. Purification of Human MEG-CSF from Hypomegakaryocytic Thrombocytopenic Plasma

\begin{tabular}{|c|c|c|c|c|c|}
\hline Purification step & Volume & $\begin{array}{l}\text { Total } \\
\text { protein }\end{array}$ & $\begin{array}{l}\text { Net megakaryocyte } \\
\text { colonies }\end{array}$ & $\begin{array}{l}\text { Net colonies/mg } \\
\text { protein }\end{array}$ & $\begin{array}{l}\text { Percent } \\
\text { recovery }\end{array}$ \\
\hline & $m l$ & $m g$ & $n$ & $n$ & \\
\hline \multicolumn{6}{|l|}{ Hypomegakaryocytic thrombocytopenic } \\
\hline plasma & 400 & 13,600 & 40,800 & 3.0 & 100 \\
\hline $80 \%$ saturated $\left(\mathrm{NH}_{4}\right)_{2} \mathrm{SO}_{4}$ fraction & & 1,370 & 18,221 & 13.3 & 44.7 \\
\hline \multicolumn{6}{|c|}{ DEAE-Sepharose CL-6B* chromatography } \\
\hline Fraction IV & & 192 & 6,720 & 35.0 & 16.5 \\
\hline Fraction V & & 31.2 & 7,982 & 256.0 & 19.6 \\
\hline Fraction VI & & 5.4 & 297 & 55.0 & 0.7 \\
\hline \multicolumn{6}{|c|}{$\begin{array}{l}\text { Wheat germ lectin-Sepharose } 6 \mathrm{MB} \text { affinity } \\
\text { chromatography }\end{array}$} \\
\hline Peak I & & 11.8 & 0 & 0 & 0.0 \\
\hline Peak II & & 5.0 & 7,500 & 1,500 & 18.4 \\
\hline RP-HPLC§ & & 0.65 & 6,802 & 10,466 & 16.6 \\
\hline
\end{tabular}

* Three individual fractions obtained by DEAE-Sepharose CL-6B column chromatography. $\ddagger$ Peak I was desorbed with starting buffer while peak II was desorbed with starting buffer containing $0.1 \mathrm{M} \mathrm{N}$-acetyl-D-glucosamine. § Experiments were carried out utilizing analytical reverse-phase high-performance liquid chromatography (RP-HPLC).

disk or slab gels unless otherwise stated. Gels containing 3 (stacking gel), 7.5 , or $10 \%$ polyacrylamide were prepared from a stock solution of $30 \%$ acrylamide by weight and $0.8 \% N, N^{\prime}$-bis-methylene acrylamide. Prior to application on the gel, the samples were heated to $100^{\circ} \mathrm{C}$ for $3 \mathrm{~min}$ in the sample buffer. Gels to be stained with Coomassie Blue and periodic acid-Schiff (PAS) were loaded with 15 and $30 \mu \mathrm{g}$ of protein, respectively. The disk gels $(5 \times 125 \mathrm{~mm})$ were run at $5 \mathrm{~mA} /$ tube until the Bromophenol Blue tracking dye had reached the lower edge of the gel. Duplicate gels were fixed and stained with Coomassie Brilliant Blue R-250 in acetic acid/methanol/water (1:5:4 by volume) or with the PAS reaction. Known molecular weight proteins $\{$ Bio-Rad
Laboratories) were simultaneously run in the gels to serve as molecular weight reference markers. Markers consisted of $5 \mu \mathrm{g}$ of myosin-H $\left(M_{\mathrm{r}}\right.$ $200,000), \beta$-galactosidase $\left(M_{\mathrm{r}} 130,000\right)$, phosphorylase-B $\left(M_{\mathrm{r}} 92,500\right)$, bovine serum albumin $\left(M_{\mathrm{r}} 66,200\right)$, ovalbumin $\left(M_{\mathrm{r}} 45,000\right)$, carbonic anhydrase $\left(M_{\mathrm{r}} 32,000\right)$, and soy bean trypsin inhibitor $\left(M_{\mathrm{r}} 20,000\right)$ $(25,26)$.

Deglycosylation by TFMSA. The partially purified material obtained after wheat germ lectin affinity chromatography was deglycosylated according to the method of Edge et al. (27). Briefly, $1 \mathrm{ml}$ of anisole and $2 \mathrm{ml}$ of TFMSA were mixed in a glass tube with a screw cap and cooled to $0^{\circ} \mathrm{C}$. The partially purified fraction $(0.6 \mathrm{mg})$ was added to

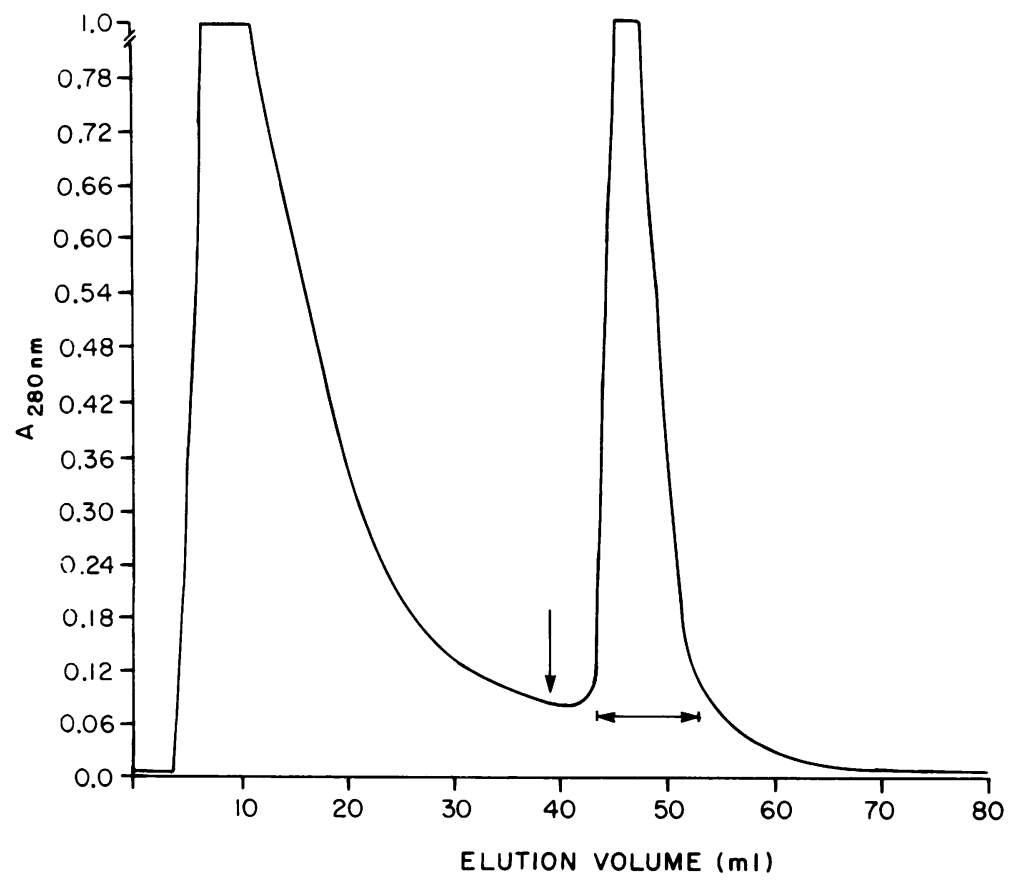

Figure 2. Elution profile of human Meg-CSF from wheat germ-lectin Sepharose beads by $N$-acetyl-glucosamine. $60 \mathrm{mg}$ of fraction $\mathrm{V}$ obtained by gel filtration column chromatography was applied in a vol of $2 \mathrm{ml}$ to a $9 \times 150$-mm column of wheat germ lectinSepharose $6 \mathrm{MB}$ equilibrated with starting buffer $(0.05$ $\mathrm{M}$ phosphate buffer, $\mathrm{pH} 7.2$, containing $0.2 \mathrm{M} \mathrm{NaCl}$ and $0.02 \%$ [wt/vol] $\mathrm{NaN}_{3}$ ). The column was eluted with starting buffer at a flow rate of $40 \mathrm{ml} / \mathrm{h}$. Fractions were collected in $1.5 \mathrm{ml} /$ tube and the absorbance at $280 \mathrm{~mm}$ was monitored. The vertical arrow indicates the application of $0.1 \mathrm{M} \mathrm{N}$-acetyl-D-glucosamine in starting buffer as a second elution. The horizontal arrow indicates the pool made. 


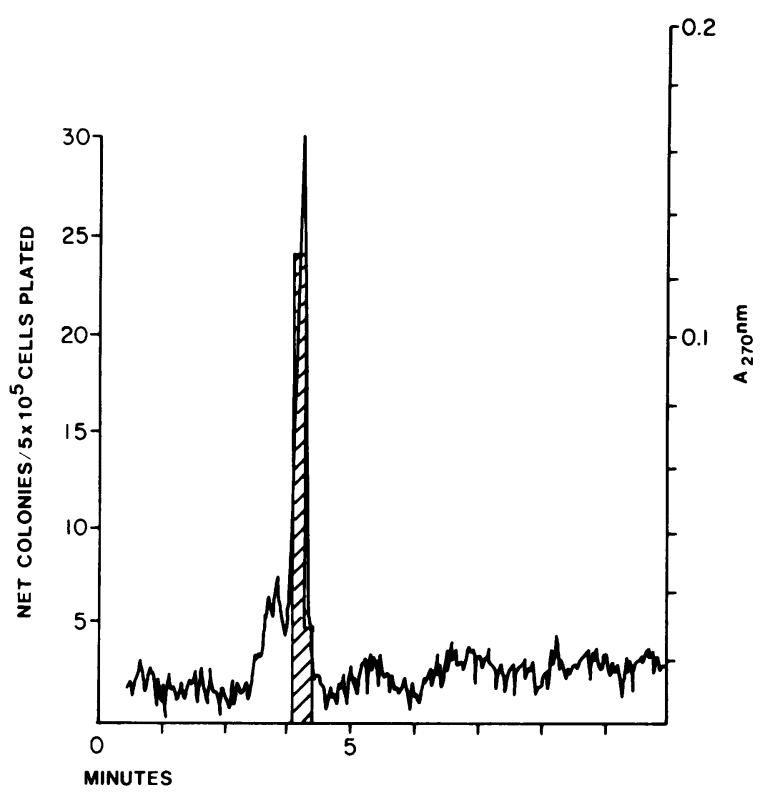

Figure 3. Reverse-phase high performance liquid chromatography of Meg-CSF. $30 \mu \mathrm{g}$ of protein was injected in a vol of $10 \mu \mathrm{l}$ into a 0.4 $\times 25-\mathrm{cm}$ column equilibrated with $30 \% \mathrm{CH}_{3} \mathrm{CN}$. The column was eluted at $0.5 \mathrm{ml} / \mathrm{min}$ with a linear gradient of $30-70 \% \mathrm{CH}_{3} \mathrm{CN}$. Fractions were collected in $0.3 \mathrm{ml} /$ tube and the absorbance at 270 $\mathrm{nm}$ was monitored; Meg-CSF activity of each fraction was quantitated by the ability of the fractions to promote megakaryocyte colony formation by normal human marrow cells and is indicated by hatched area (IIIII); the solid line (-) indicates the absorbance of the collected fractions.

this precooled mixture $(0.6 \mathrm{ml})$ and the reaction was allowed to proceed at room temperature for $40 \mathrm{~min}$. Then $6 \mathrm{ml}$ of diethyl ether (precooled in frozen $\mathrm{CO}_{2}$ ) was added, followed by $1 \mathrm{ml}$ of cold $50 \%$

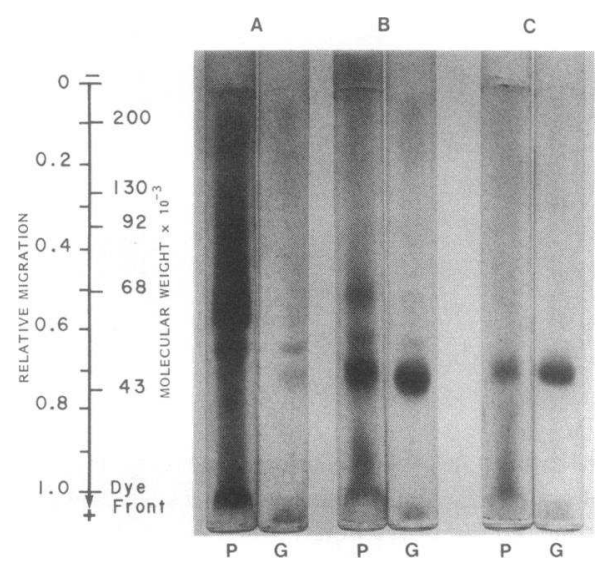

Figure 4. SDS-polyacrylamide electrophoretic gel analysis of the most active fractions from each step of the purification scheme of MegCSF from human hypomegakaryocytic thrombocytopenic plasma. $(A)$ $80 \%$ saturated ammonium sulfate precipitate. $(B)$ Fraction $V$ from ion exchange column chromatography on DEAE-Sepharose CL-6B. (C) Fraction desorbed from the wheat germ lectin-Sepharose $6 \mathrm{MB}$ by $\mathrm{N}$-acetyl-D-glucosamine. P, protein pattern as indicated by Coomassie Blue staining; G, glycoprotein pattern as indicated by PAS staining. The relationship between relative migration and molecular weight is shown at left. (vol/vol) aqueous pyridine. The solution was centrifuged, after which the ether phase was removed with a pasteur pipette. This extraction procedure was repeated another time. The remaining aqueous-phase volumes were combined and diluted to total volume of $2 \mathrm{ml}$ with water, and pyridine was removed by gel filtration on P-6DG column (Bio-Rad Laboratories) equilibrated with PBS. The void volume fractions were collected in a final volume of $5.6 \mathrm{ml}$ and concentrated to dryness in a Speed Vac concentrator. The dried material was stored at $-20^{\circ} \mathrm{C}$ before assay for Meg-CSF activity. At the time of assay the dried fractions were suspended in $\alpha$-medium at various protein concentrations.

\section{Results}

Purification. When bone marrow cells are cloned in the absence of a source of Meg-CSF, 14.6 \pm 8.5 (mean \pm 1 SD) megakaryocyte colonies per $5 \times 10^{5}$ marrow cells plated were observed. This background colony formation was observed in each of the 10 normal marrow samples studied. After ammonium sulfate fractionation at $60 \%$ saturation, no Meg-CSA activity was detected in the precipitate. A small amount of activity was recovered in the $70 \%$ fraction (data not shown). The greatest activity was recovered in the precipitate which formed at $80 \%$ saturation (Table I).

The elution profile resulting from DEAE-Sepharose CL6B chromatography of the $80 \%\left(\mathrm{NH}_{4}\right)_{2} \mathrm{SO}_{4}$ fraction is shown in Fig. 1. A significant amount of protein was eliminated in peaks II and III, which emerged before the active fractions, peaks IV, V, and VI. The most potent Meg-CSF activity resided in fraction $\mathrm{V}$ (Table $\mathrm{I}$ ).

Further purification of the protein responsible for megakaryocyte colony formation was achieved after wheat germ lectin-affinity chromatography (Fig. 2). No activity was detected in the eluate desorbed with the starting buffer. In contrast, a highly purified fraction was obtained following batch elution of the column with $\mathrm{N}$-acetyl-D-glucosamine (Table I).

Fig. -3 illustrates the elution profile as obtained from a Whatman C18 ODS3 column (Whatman, Inc., Clifton, NJ) by reverse-phase high performance liquid chromatography. The major peak of Meg-CSF activity coincided with the main peak of ultraviolet absorbing material. The highly purified material was capable of promoting the formation of 10,466 megakaryocyte colonies/mg of protein (Table I). The four-step purification scheme resulted in the recovery of $16.6 \%$ of the assayable Meg-CSF present in the starting plasma specimen.

Apparent molecular weight. The molecular weight of MegCSF was determined by its relative migration in comparison to known molecular weight markers in SDS-polyacrylamide gels. Duplicate gels stained with Coomassie Blue and PAS are shown in Fig. 4. The material that eluted off the wheat germ lectin-affinity column produced a single band whose staining characteristics were consistent with its being a glycoprotein with an apparent mol wt of 46,000 (Fig. 4). The electrophoretic mobility of the purified Meg-CSF in SDS-PAGE under reduced and nonreduced conditions are identical. It is possible that the true molecular weight based on amino acid and carbohydrate analysis may be lower due to the aberrant migration of glycoproteins in SDS-polyacrylamide gels $(25,26)$. The relative migration of purified Meg-CSF and a preparation of partially purified human urinary Epo in SDS-polyacrylamide gels is illustrated in Fig. 5. As can be seen, based upon this parameter, these two glycoproteins are distinct moieties. The Coomassie 


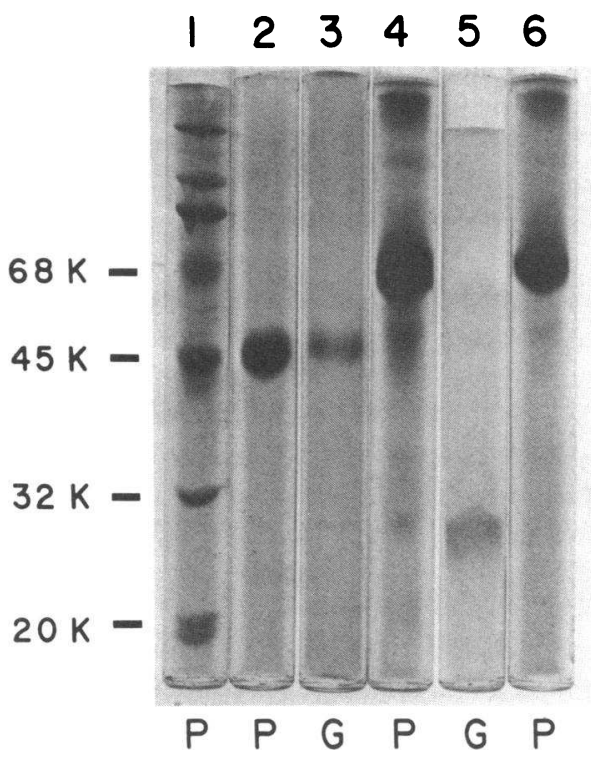

Figure 5. Electrophoretogram of purified Meg-CSF and erythropoietin on a $10 \%$ SDS-polyacrylamide gel. Lane 1 , standard molecular weight markers $(2.5 \mu \mathrm{g})$; lanes 2 and 3, purified Meg-CSF from human hypomegakaryocytic thrombocytopenic plasma $(50 \mu \mathrm{g})$; lanes 4 and 5 , purified human urinary erythropoietin $(10 \mathrm{U}$, sp act 500 $\mathrm{U} / \mathrm{mg}$ of protein); lane 6 , bovine serum albumin alone. In lane 4 , the heavily stained band represents bovine serum albumin which had been used by the Alpha Therapeutic Corp. to preserve the biological activity of Epo. P, protein pattern as indicated by Coomassie Blue staining; $\mathrm{G}$, glycoprotein pattern as indicated by PAS staining.

Blue and PAS staining bands seen in lanes 4 and 5 of Fig. 5 are indicative of a glycoprotein with an apparent mol wt of $\sim 30,000$, which is presumed to represent Epo.

Importance of oligosaccharide component for Meg-CSF activity. Edge et al. (27) have reported that the treatment of glycoproteins with TFMSA cleaves oligosaccharides from the glycoproteins without cleaving the protein core. Therefore the activity of a purified fraction was tested before and after the addition of TFMSA. Deglycosylation of Meg-CSF by this method abolished the ability of the purified fraction to promote megakaryocyte colony formation (Table II).

Stimulation of colony formation by bone marrow cells. The effect of varying concentrations of purified Meg-CSF on megakaryocyte colony formation is shown in Fig. 6 . As can be seen in this figure, there appears initially to be a linear relationship between the dose of Meg-CSF and the number of colonies enumerated. At higher concentrations, however, the effect of Meg-CSF appears to reach a plateau. At a concentration of

Table II. Effect of TFMS Treatment on MEG-CSF Activity

\begin{tabular}{ll}
\hline Addition to culture & $\begin{array}{l}\text { Mean no. of colonies per } \\
5 \times 10^{5} \text { cells plated }\end{array}$ \\
\hline None & 12.5 \\
Meg-CSF & 32.5 \\
TFMS-treated Meg-CSF & 7.8 \\
\hline
\end{tabular}

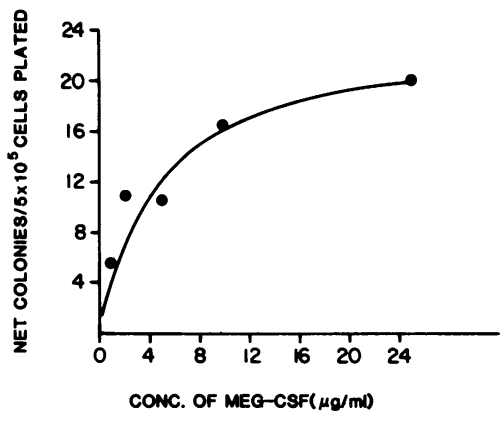

Figure 6. Effect of varying concentrations of purified Meg-CSF on megakaryocyte colony formation by normal human marrow cell. Each point represents the mean of assays performed in quadruplicate.

$7.6 \times 10^{-8} \mathrm{M}$, the purified Meg-CSF was capable of promoting the formation of one-half of the maximal numbers of megakaryocyte colonies by normal bone marrow cells.

In Table III the specificity of the action of purified MegCSF is shown. The ability of Meg-CSF to promote CFU-Eand BFU-E-derived colonies is compared with that of a source of Epo, and its ability to promote CFU-GM-derived colonies is compared with a PHA-LCM with a known granulocytemacrophage colony-stimulating activity (GM-CSA). From this data it is evident that the purified Meg-CSF does not have the ability to promote CFU-GM, BFU-E-, or CFU-E-derived colony formation. In addition, in Table III it is shown that the 46,000 mol wt band when eluted from the SDS-PAGE retained $75 \%$ of its biological activity. This data further substantiates our contention that this glycoprotein is the putative Meg-CSF. We have previously suggested that Meg-CSF not only alters cloning efficiency of the colony-forming unit megakaryocyte (CFU-M) but that it also increases the number of cells composing megakaryocyte colonies (17). A similar phenomena was observed when CFU-M was cloned in the presence of purified Meg-CSF. We were able to enumerate the numbers of cells in 35 colonies cloned in the absence of Meg-CSF $(9.5 \pm 3.1$ megakaryocytes [mean \pm 1 SD]). By contrast, when 37 colonies cloned in the presence of purified Meg-CSF were analyzed,

Table III. Effect of Purified MEG-CSF on Hematopoietic Colony Formation

\begin{tabular}{lllll}
\hline & \multicolumn{4}{l}{ Colonies per $5 \times 10^{5}$ cells plated } \\
\cline { 2 - 5 } Addition to culture & CFU-M & CFU-E & BFU-E & CFU-GM \\
\hline None & 9.5 & 34.0 & 1.0 & 14.5 \\
PHA-LCM* & NA" & NA & NA & 54.5 \\
Erythropoietin & NA & 558.0 & 179.0 & NA \\
Meg-CSF $1 \ddagger$ & 33.5 & 33.0 & 5.0 & 20.0 \\
Meg-CSF $\$$ & 27.0 & 3.0 & 2.5 & 22.0 \\
\hline
\end{tabular}

* Phytohemagglutinin-stimulated leukocyte-conditioned media. $¥$ Meg-CSF $_{1}$-purified Meg-CSF obtained after wheat germ lectin-affinity chromatography.

$\S \mathrm{Meg} \mathrm{CSF}_{2}$-purified megakaryocyte-stimulating factor eluted from

SDS-PAGE when Meg-CSF was applied to gel.

"NA, not available. 
they were shown to contain $16.8 \pm 4.1$ megakaryocytes (mean \pm 1 SD) per colony.

Some megakaryocyte colonies consist exclusively of or contain small fluorescein-labeled cells approximately the size of lymphocytes that are not otherwise morphologically recognizable as megakaryocytes. By contrast, other megakaryocyte colonies contain morphologically identifiable megakaryocytes. In the absence of a source of Meg-CSF, 35-75\% of all the megakaryocyte colonies present consisted exclusively of the small fluorescein-labeled cells. We have previously reported that the addition of aplastic anemia sera resulted in a relative decrease in the numbers of the colonies composed exclusively of small fluorescein positive cells (14). By contrast, the addition of purified Meg-CSF did not result in a similar increase in the numbers of colonies composed of morphologically identifiable megakaryocytes or a decrease in the numbers of colonies containing exclusively fluorescein positive small mononuclear cells.

\section{Discussion}

Factors that control human thrombopoiesis and megakaryocytopoiesis have not been well characterized (28). Thrombopoieticstimulating factor (TSF) or thrombopoietin appears to accelerate thrombopoiesis in vivo. TSF has been detected in media conditioned by human embryonic kidney cells (29) and in plasma of thrombocytopenic humans and animals $(30,31)$. Recently, a number of in vitro clonal culture systems have been developed to analyze the early events in human megakaryocytopoiesis (7, 9); Meg-CSF activity has been detected in a variety of crude human body fluids including urine $(5,6)$, serum (7), and plasma from thrombocytopenic individuals (7, 8). Meg-CSF not only increases cloning efficiency, but may also increase the proliferative capacity of individual CFU-M as evidenced by a statistically significant increase in the number of cells observed per colony (7). The variations in Meg-CSF levels that have been detected in different disease states suggest that alterations in the production of this regulator are physiologically important (7).

The availability of purified regulatory molecules and cell populations are a prerequisite for unraveling the complexities of human megakaryocytopoiesis. Such an approach has been extremely useful in the study of human and murine granulopoiesis and erythropoiesis $(32,33)$ where purification of Epo (32), erythropoietic burst-promoting activity $(34,35)$, and various subclasses of GM-CSA (36-38) have provided a better understanding of both granulopoiesis and erythropoiesis.

We approached the purification of human Meg-CSF with a four-step scheme including ammonium sulfate fractionation, ion exchange column chromatography, wheat germ lectinaffinity chromatography, and reverse-phase high performance liquid chromatography. The plasma clot culture system for human megakaryocyte colonies was utilized to assay for MegCSF activity (7). Meg-CSF activity was quantitated by the ability of a given fraction to promote megakaryocyte colony formation by normal human marrow cells. This purification scheme resulted in the isolation of a protein from the plasma of a patient with hypomegakaryocytic thrombocytopenia that we call Meg-CSF, in that it is capable of stimulating the formation of 10,466 megakaryocyte colonies/mg of protein. The yield of the active material was $16.6 \%$ and the scheme resulted in a 3,489-fold purification from plasma. The purified Meg-CSF also has the ability to increase the number of megakaryocytes composing individual megakaryocyte colonies. We have previously reported that the addition of crude aplastic anemia serum also resulted in a relative increase in the numbers of colonies composed of morphologically identifiable megakaryocytes and a decrease in the numbers of colonies containing exclusively small fluorescein-labeled cells approximately the size of lymphocytes that were not otherwise morphologically recognizable as megakaryocytes (14). These lymphocyte-like cells are thought to represent immature megakaryocytic elements (17). Interestingly, the purified Meg-CSF when added to our assay system did not result in an increase in the number of colonies composed of morphologically identifiable megakaryocytes. This finding suggests the presence of an additional factor in aplastic anemia serum that influences terminal maturation of megakaryocytes. The action of MegCSF and this maturation factor appear to be distinct. Recently, Straneva and co-workers (39) have described an assay system for such a factor present in human plasma and have termed this factor megakaryocyte maturation factor.

Coomassie Blue and PAS staining, as well as its affinity for wheat germ lectin, suggest that Meg-CSF is a glycoprotein. We have provided some evidence that the carbohydrate moiety of Meg-CSF is necessary for its activity, inasmuch as the ability of the protein to support megakaryocyte colony formation is lost upon exposure to TFMSA. TFMSA cleaves oligosaccharides from glycoproteins without damaging their protein core (19). Interestingly, Bazill and co-workers (40) recently demonstrated that the integrity of the carbohydrate moiety of a factor purified from WEHI-3 cell-conditioned media, which stimulates the proliferation and development of normal multipotent hematopoietic cells as well as megakaryocytic, erythroid, and granulocytic committed progenitor cells, was also necessary for it to be able to promote colony formation.

The molecular weight of purified Meg-CSF was estimated to be 46,000 by SDS-PAGE. This molecular weight determination was very similar to the findings of Kawakita et al. (6) for Meg-CSF activity detected in the urinary extracts of patients with aplastic anemia. These investigators found two distinct peaks of activity after Sephadex G-200 gel filtration, which were estimated to have apparent mol wt of 155,000 and 76,000 . When gel filtration was perform under disaggregating conditions (6 M guanidine hydrochloride), the first peak was significantly reduced and a single peak with an apparent mol wt of 45,000 was observed.

Several groups have reported that Epo is capable of stimulating megakaryocyte colony formation in vitro $(5,41)$. It appears likely that our Meg-CSF activity is distinct from Epo. Asialo-erythropoietin and native Epo have apparent mol wt on SDS-PAGE of 34,000 and 39,000, respectively (32); however, human Meg-CSF has an apparent mol wt of 46,000 on SDSPAGE. In addition, our purified Meg-CSF was unable to support erythroid colony formation by normal human marrow cells. That Meg-CSF and Epo are different moieties has been previously substantiated by the fact that the ability of aplastic anemia serum to support erythroid but not megakaryocyte colony formation can be abolished by prior treatment of the serum with an antierythropoietin antisera (42). The relationship between the purified Meg-CSF and thrombopoietin is as yet undetermined. These studies will be particularly difficult, be- 
cause an adequate in vitro assay system for thrombopoietin is not presently available.

The exact cellular target of Meg-CSF is not presently known. Unfractionated human marrow mononuclear cell preparations were used in the present assay system as target cells. The importance of cellular interactions in the regulation of hematopoiesis is well established (43). Bagby and coworkers, for example, have recently shown that GM-CSA can be produced by vascular endothelial cells in response to a stimulating monokine (44). Whether Meg-CSF directly affects the CFU-M or exerts its effect through such a marrow accessory cell pathway is yet to be determined. Whatever its exact mechanism of action might be, the biological effect of MegCSF appears to be rather specific in that it does not increase cloning efficiency of other progenitor cells.

Purification of human Meg-CSF from hypomegakaryocytic plasma has potential clinical significance. The presence of varying amounts of this factor in urine, plasma, and serum of patients with disorders of megakaryocytopoiesis indicates its physiological importance in the regulation of megakaryocytopoiesis. The purification of this molecule will allow a more direct analysis of the cellular and molecular events in human megakaryocytopoiesis. In addition, the feasibility of the administration of large quantities of this purified protein to patients with thrombocytopenia should be explored.

\section{Acknowledgments}

We would like to extend our thanks to Ms. Linda Cheung and Cynthia Booth for their excellent secretarial support, and to Asok Antony, M.D., and Hal Broxmeyer, Ph.D., for helpful discussions.

This study was supported in part by U. S. Public Health Service grant CA-34841. Dr. Hoffman is a recipient of a Research Career Development Award from the U. S. Public Health Service.

\section{References}

1. Williams, N., R. R. Eger, H. M. Jackson, and D. J. Nelson. 1982. Two factor requirement for murine megakaryocyte colony formation. J. Cell. Physiol. 110:101-104.

2. Williams, N., and R. F. Levine. 1982. The origin, development and regulation of megakaryocytes. Br. J. Haematol. 52:173-179.

3. Iscove, N. N., C. A. Roitsch, N. Williams, and L. J. Guilbert. 1982. Molecules stimulating early red cell, granulocyte, macrophage and megakaryocyte precursors in culture: similarity in size, hydrophobicity and charge. J. Cell. Physiol. Suppl. 1:67-78.

4. Bertoncello, I., T. R. Bradley, and G. S. Hodgson. 1981. Characterization and enrichment of macrophage progenitor cells from normal and 5-fluorouracil treated mouse bone marrow by unit gravity sedimentation. Exp. Hematol. 9:604-610.

5. Enomoto, K., M. Kawakita, S. Kishimoto, N. Katayuma, and T. Miyake. 1980. Thrombopoiesis and megakaryocyte colony stimulating factor in the urine of patients with aplastic anemia. Br. J. Haematol. 45:551-556.

6. Kawakita, M., T. Miyake, S. Kishimoto, and M. Ogawa. 1982. Apparent heterogeneity of human megakaryocyte colony and thrombopoiesis-stimulating factors: studies on urinary extracts from patients with aplastic anemia and idiopathic thrombocytopenic purpura. $B r . J$. Haematol. 52:429-438.

7. Hoffman, R., E. Mazur, E. Bruno, and V. Floyd. 1981. Assay of an activity in the serum of patients with disorders of thrombopoiesis that stimulates formation of megakaryocyte colonies. N. Engl. J. Med. 305:533-538.
8. Messner, H. A., N. Jamal, and C. Izaguirre. 1982. The growth of large megakaryocyte colonies from human bone marrow. J. Cell. Physiol. Suppl. 1:45-51.

9. Kimura, H., S. A. Burstein, D. Thorniny, J. S. Powell, L. A. Harker, P. J. Fialkow, and J. W. Adamson. 1984. Human megakaryocytic progenitors (CFU-M) assaying in methylcellulose: physical characteristics and requirements for growth. J. Cell. Physiol. 118:8796.

10. Williams, N., H. Jackson, P. Ralph, and I. Nakoinz. 1981. Cell interactions influencing murine marrow megakaryocytopoiesis: nature of the potentiator cell in bone marrow. Blood. 57:157-163.

11. Vainchenker, W., J. Chapman, J. F. Deschamps, G. Vinci, J. Bouget, M. Titeux, and J. Breton-Gorius. 1982. Normal human serum contains a factor capable of inhibitory megakaryocyte colony formation. Exp. Hematol. 10:650-656.

12. Stoll, D. B., S. Blum, D. Pasquale, and S. Murphy. 1981. Thrombocytopenia with decreased megakaryocytes. Ann. Intern. Med. 94:170-175.

13. Hoffman, R., E. Bruno, J. Elwell, E. Mazur, A. M. Gewirtz, P. Dekker, and A. E. Denes. 1982. Acquired amegakaryocytic thrombocytopenic purpura: a syndrome of diverse etiologies. Blood. 60:11731178.

14. Mazur, E., R. Hoffman, and E. Bruno. 1981. Regulation of human megakaryocytopoiesis: an in vitro analysis. J. Clin. Invest. 68: 733-741.

15. McLeod, D. L., M. M. Shreeve, and A. A. Axelrod. 1976. Induction of megakaryocyte colonies with platelet formation in vitro. Nature (Lond.). 261:492-494.

16. Marchesi, S. L., and J. A. Chasis. 1979. Isolation of human platelet glycoproteins. Biochim. Biophys. Acta. 555:442-459.

17. Mazur, E., R. Hoffman, S. Marchesi, J. Chasis, and E. Bruno. 1981. Immunofluorescent identification of human megakaryocyte colonies using an antiplatelet glycoprotein antiserum. Blood. 57:277-286.

18. Ritchey, A. K., R. Hoffman, E. Coupal, V. Floyd, H. A. Pearson, and B. G. Forget. 1981. Imbalanced globin chain synthesis in cultured erythroid progenitor cells from thalassemic bone marrow and peripheral blood. Blood. 57:788-793.

19. McLeod, D. L., M. M. Shreeve, and A. A. Axelrad. 1974 Improved plasma culture system for production of erythrocytic colonies in vitro quantitative assay method for CFU-E. Blood. 44:517-534.

20. Fauser, A. A., and H. A. Messner. 1978. Granuloerythropoietic colonies in human bone marrow, peripheral blood and cord blood. Blood. 52:1243-1248.

21. Aye, M. T., Y. Niho, J. E. Till, and E. H. McCulloch. 1974 Studies of leukemic cell populations in culture. Blood. 44:205-219.

22. Lowry, O. H., N. J. Rosebrough, A. L. Farr, and R. J. Randall. 1951. Protein measurement with the Folin phenol reagent. J. Biol Chem. 193:265-272.

23. March, S. C., I. Parikh, and P. Cuatrecasas. 1974. A simplified method for cyanogen bromide activation of agarose for affinity chromatography. Anal. Biochem. 60:149.

24. Laemmli, U. K. 1980. Cleavage of structure proteins during the assembly of the head of bacteriophage $\mathrm{T}_{4}$. Nature (Lond.). 227: 680-685.

25. Allen, R. H., and P. W. Majerus. 1972. Isolation of vitamin $\mathrm{B}_{12}$-binding proteins using affinity chromatography. J. Biol. Chem. 247:7702-7708.

26. Andrews, P. 1965. The gel filtration behavior of proteins related to their molecular weights over a wide range. Biochem. J. 96:595-606.

27. Edge, A. S. B., C. R. Faltynev, L. Hof, L. E. Reichart, and P. Weber. 1981. Deglycosylation of glycoproteins by trifluoromethanesulfonic acid. Anal. Biochem. 118:131-137.

28. Levin, J., and B. L. Evatt. 1979. Humoral control of thrombopoiesis. Blood Cells. 5:105-121.

29. McDonald, T. P., R. Clift, and R. D. Lange. 1975. Thrombopoietin production by human embryonic kidney cells in culture. $J$. Lab. Clin. Med. 85:59-66. 
30. Evatt, B. L., D. P. Shreiner, and J. Levin. 1974. Thrombopoietic activity of fractions of rabbit plasma studies in rabbits and mice. $J$. Lab. Clin. Med. 83:364-371.

31. Shreiner, D. P., P. J. Weinberg, and D. Enoch. 1980. Plasma thrombopoietic activity in humans with normal and abnormal platelet counts. Blood. 56:183-188.

32. Miyake, T., C. K. H. Kung, and E. Goldlwasser. 1977. Purification of human erythropoietin. J. Biol. Chem. 252:5558-5564.

33. Metcalf, D., and N. A. Nicola. 1983. Proliferative effects of purified granulocyte colony-stimulating factor (G-CSF) on normal mouse hemopoietic cells. J. Cell. Physiol. 116:198-206.

34. Porter, P. N., and M. Ogawa. 1982. Characterization of human erythroid burst-promoting activity derived from bone marrow conditioned media. Blood. 59:1207-1212.

35. Westbrook, C., J. Gassen, M. Selsted, and D. W. Golde. 1983. Purification of a human T-lymphocyte-derived burst promoting activity. Blood 62(Suppl. 1):417. (Abstr.)

36. Burgess, A. W., J. Camakaris, and D. Metcalf. 1977. Purification and properties of colony stimulating factor from mouse lung-conditioned medium. J. Biol. Chem. 252:1998-2203.

37. Waheed, A., and R. K. Shadduck. 1982. Purification of colony stimulating factor by affinity chromatography. Blood. 60:238-244.

38. Stanley, E. R., and P. M. Heard. 1977. Factors regulating macrophage production and growth: purification and some properties of the colony stimulating factor from medium conditioned by mouse L cells. J. Biol. Chem. 252:4305-4312.

39. Straneva, J., E. Bruno, G. Beyer, A. Floyd, and R. Hoffman. 1984. Detection of factors that regulate cytoplasmic and nuclear maturation of human megakaryocytes. Clin. Res. 32:324A

40. Bazill, G. W., M. Haynes, J. Garland, and T. M. Dexter. 1983. Characterization and partial purification of a heamopoietic cell growth factor in WEHI-3 cell conditioned medium. Biochem. J. 210:747-759.

41. Vainchenker, W. J., J. Quichard, and J. Breton-Gorius. 1979. Growth of human megakaryocyte colonies in culture from human bone marrow prescursors. Blood. 54:940-945.

42. Hoffman, R., E. Mazur, A. M. Gewirtz, J. Straneva, E. Bruno, and R. Berkow. 1983. Cellular and humoral regulation of human megakaryocytopoiesis. In Normal and Neoplastic Hematopoiesis. D. W. Golde, and P. A. Marks, editors. Alan R. Liss, Inc., New York. 269-301.

43. Dexter, T. M., T. D. Allen, and L. G. Lajtha. 1977. Conditions controlling the proliferation of haemopoietic stem cells in vitro. $J$. Cell. Physiol. 91:334-335.

44. Bagby, G. C. Jr., E. McCall, K. A. Bergstrom, and D. Berger. 1983. A monokine regulators colony stimulating activity production by vascular endothelial cells. Blood. 62:663-668. 